\title{
VII. \\ Ueber die histologische Veränderung des Muskelgewebes bei der Lepra und eine besondere Wucherung und Hyperchromatose der Muskelkerne.
}

(Beiträge zur pathologischen Anatomie des quergestreiften Muskels.)

(Aus dem Pathologischen Institut in Strassburg i. E.)

Von

Dr. med. Akira Fujinami aus Japan.

(Hierzu Tafel V.)

Ueber die Veränderung des Muskelgewebes in der Lepra ist bis jetzt nur sehr wenig geschrieben worden. In den pathologischanatomischen Schilderungen dieser Krankheit, welche, soweit sie das Muskelgewebe betreffen, noch dürftig zu nennen sind, ist höchstens von einer Atrophie der Muskelprimitivbündel und einer interstitiellen Myositis die Rede. Thoma ${ }^{1}$ ) hat sogar die Immunität der Musculatur gegenüber der leprösen Erkrankung betont.

Neuerdings hat nun Herr Prof. von Recklinghausen ${ }^{2}$ ) in der Sitzung der deutschen pathologischen Gesellschaft einen Fall von Lepra tuberosa, die in Tonkin (China) erworben wurde, demonstrirt, und besonders auf die Veränderung des Muskelgewebes ("lepröse Perimyositis“) hingewiesen. Mein hochverehrter Lehrer hat alsdann die Güte gehabt, mir dieses Material zur weiteren Durchforschung zu übergeben, wofür ich mich zu besonderem Dank verpflichtet fühle.

1) Thoma: Beiträge zur pathologischen Anatomie der Lepra arabum. Dieses Archiv, Bd. 57, S. 455, 1873.

2) Verbandlungen der Deutschen Patbologischen Gesellschaft. I. Tagung (gebalten zu Düsseldorf den 19. September 1898) Berlin, 1899, S. 9. Verhandlungen der Gesellschaft deutscher Naturforscher und Aerzte. 70. Versammlung zu Düsseldorf. II. Theil, II. Hälfte. S. 5-6. Leipzig. 1899. 

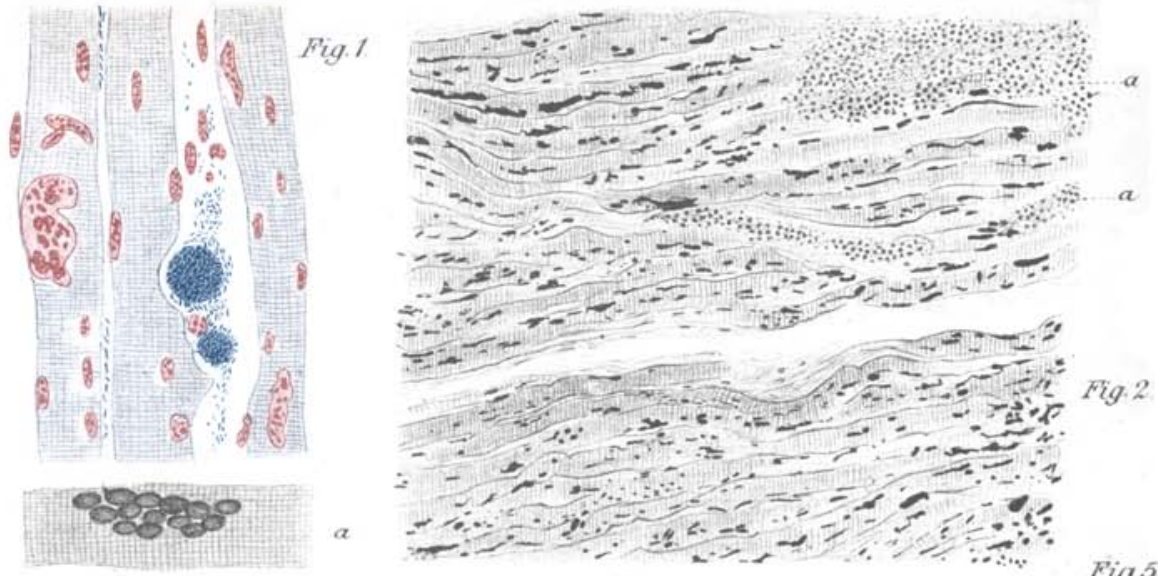

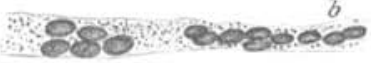

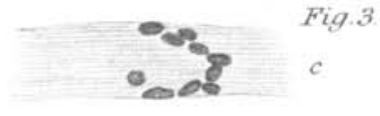

Fig. 3

\section{6.}

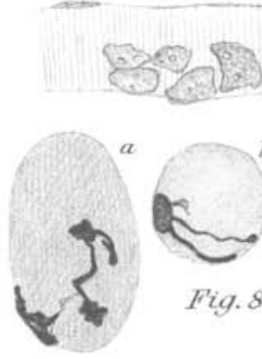

जing Fig. 4 .
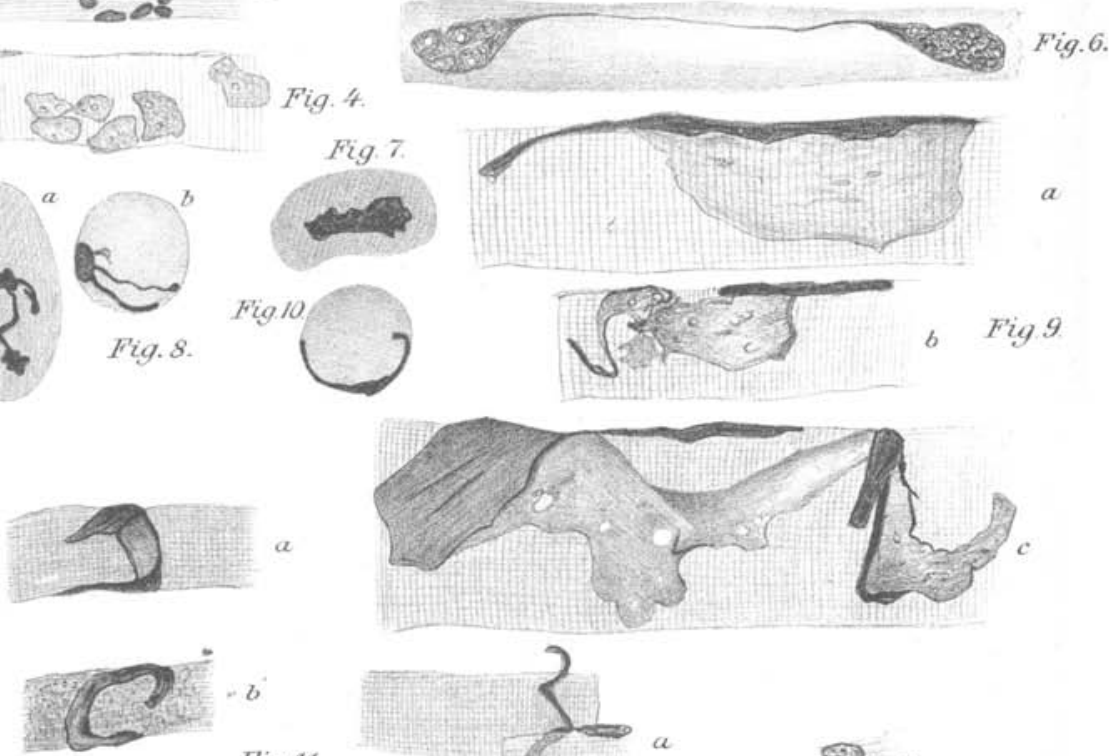

Fig11
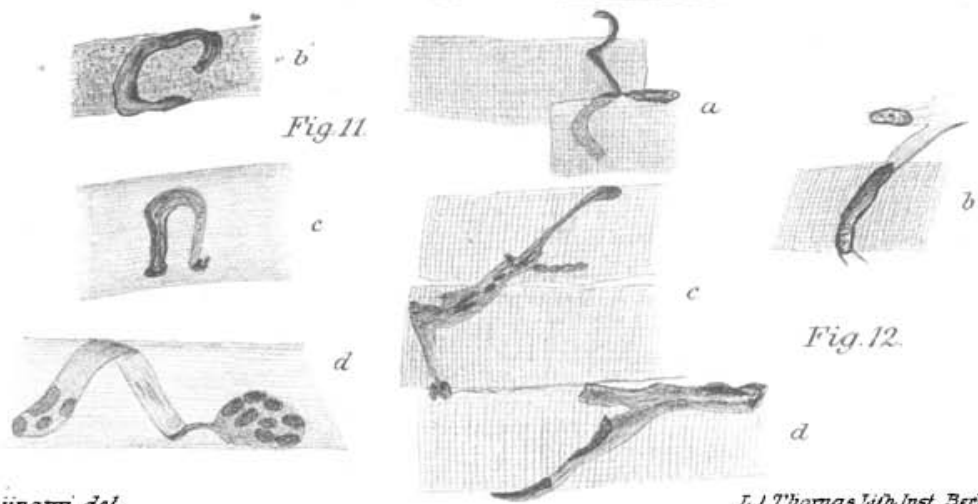
Schon makroskopisch fallen, wie von Recklinghausen schildert, weisse feine Längsstreifen auf, welche sich zwischen den Maskelbündeln befinden und auch am in Formalin gehärteten Präparate noch sehr gut zu erkennen sind. Diese weissen Längsstreifen erscheinen am deatlichsten und zahlreichsten an den Muskeln des Oberschenkels und des Oberarms, und zwar in den direct unterhalb der leprösen Heerde der Hand gelegenen Muskelabschnitten. Was ibre histologische Structur betrifft, so besteht in ihnen stellenweise eine interstitielle lipomatose, meistentheils und in sehr auffallender Weise aber eine beträchtliche Infiltration mit rundlichen Zellen $z$ wischen den gleichmässig oder nngleichmässig atrophirten Muskelprimitivbündeln, wie sie sonst an dem leprösen Heerde beobachtet wird. Die Kerne solcher infiltrirenden Zellen sind entweder ganz rund oder von zackiger, unregelmässiger Gestalt. Neben diesen Zellen und auch dort, wo dieselben feblen, ist eine grob- und feinkörnige, bröckelige Masse zwischen den atrophischen Muskelprimitivbündeln, bezw. in den Spalten des interstitiellen Gewebes angehäuft. Dass diese körnige Masse meist fettiger Natur ist, hat schon von Recklinghausen gezeigt; es sind darin auch in geringer Anzahl bräunliche Pigmentkörner nachzuweisen, die gewiss den veränderten Muskelprimitivbündeln entstammen; in einigen Stellen konnte ich einen deutlichen Uebergang zu einem weniger veründerten Muskelprimitivbündel verfolgen.

Die Leprabacillen sind im Muskelgewebe gewöhnlich nicht nachzuweisen. Nar unterhalb der leprösen Heerde der Haut sind typische Leprazellen, bezw. Colonien der Leprabacillen, wenn auch in wenigen Stellen, hier aber ziemlich reichlich vorhanden. Mit Ziehl'scher Lösung, noch schöner nach der Gram-Günther'schen Methode und auch mit Safranin-Jod ist es mir leicht gelungen, die Bakterien zu färben (Fig. 1). Gewöhnlich haben die Bacillen ihren Bitz zwischen den atrophischen Muskelprimitivbündeln und mitten in der oben erwähnten körnigen Masse. Sie stellen sich meist als zugespitzte Stäbchen dar, und sind entweder diffns gefärbt oder körnig; sogenannte metachromatische Körperchen sind häufig sehr deutlich nachzuweisen, so dass die Bacillen bei flüchtiger Beobachtung wie einfache Kokkenketten aussehen. Was den Sitz der Bacillen 
anbetrifft, so konnte ich bezüglich der getheilten Ansichten der Autoren - einerseits extracellulär (Unna und seine Schüler), andererseits intracellulär (Neisser, Hansen, Cornil und Suchard u. s. w.) - der Mehrzahl der Pathologen und Bakteriologen zustimmen, denn ich konnte in manchen Fällen die Leprabacillen oder deren Colonien innerhalb der Zellen, mochten es nun Leukocyten, Bindegewebszellen oder Plasmazellen sein, deutlich nachweisen. Nicht selten befanden sich dicht bei der Bacillencolonie innerhalb einer Zelle ein, zwei oder noch mehr Kerne, deren Gestalt durch Bacillenhaufen beeinflusst zu sein schien; manchmal fehlte dagegen der Kern neben dem Bacillenhaufen. Dass die Leprabacillen sich in den Spalträumen des Interstitiums auch ausserhalb der Zellen zu Colonien, welche dem Aussehen nach den "Leprazellen " äusserst ähnliche, rundliche Klumpen darstellen, auswachsen können, halte ich mit Babes für sehr wahrscheinlich. Häufig sind die Colonien oder Zooglöen der Leprabacillen mit grösseren oder kleineren rundlichen, blassgefärbten und homogen aussehenden Räumen versehen, die man auch sonst nicht selten an den Leprabacillen-Haufen trifft. An wenigen Stellen sab ich in dem stark gewucherten Intima-Gewebe, sogar selbst in den Endothelzellen einer kleineren Arterie zahlreiche Leprazellen, bezw. Bacillencolonien. Vereinzelte Leprabacillen sind sowohl in fixen Bindegewebszellen, Leukocyten, Endothelzellen der Capillargefüsse, als auch in den Gewebsspalten und im Gefässlumen vorhanden. Innerhalb der Kerne wurden die Bacillen erst gefunden, wenn jene in Verblassung und Aufquellung oder sogar in Auflösung gerathen waren; jedenfalls ein seltener Befund. In den intermusculär gelegenen kleinen Nervenfasern waren Leprabacillen nicht nachzuweisen. Innerhalb der wohl erhaltenen Muskelprimitivbündel habe ich niemals Leprabacillen gesehen; nur in den stark körnig zerfallenen Muskelprimitivbündeln befinden sich ab und zu vereinzelte Leprabacillen.

Es ist jetzt nicht meine Hauptaufgabe, hier in eine Discussion über die Leprabacillen einzutreten, da dem Befunde der letzteren im Muskelgewebe nur eine secundäre Bedeatung zuzukommen und eine directe Fortplanzung von dem oberflächlichen Heerde her vorzuliegen scheint. 
Ein viel grösseres Interesse knüpft sich an die Veränderung der Muskelprimitivbündel, besonders ihrer Kerne.

Die Muskelprimitivbündel sind im Allgemeinen atrophisch, wie manche Autoren in dem leprösen Muskel beobachtet and beschrieben baben; die Querstreifung ist nicht immer gut erhalten, erscheint vielmehr besonders an den stark atrophisehen Muskelprimitivbundeln bald mehr, bald weniger verwaschen. Dieselben zeigen nehmlich entweder eine sehr unregelmässige Anordnung der Querstreifung, oder au ihrer Stelle eine körnige und pigmentäre Entartang. Also handelt es sich nicht um oine ächte einfache Atrophie. Diese Veränderung tritt gewöhnlich gleichmässig in der ganzen Breite der Muskelprimitivbündel auf; ihr Contour erscheint dabei gewöhnlich glatt, manchmal aber auch uneben, besonders an stärker atrophirten Muskelprimitivbündeln. Ich sah diese Veränderung immer am beträchtlichsten in directer Nähe des leprösen Heerdes der Haut, dagegen tritt sie in den weit davon entlegenen Stellen nur ganz gering oder gar nicht zu Tage. Nicht selten fallen einige Muskelprimitivbündel, verglichen mit den benachbarten, durch ihre grosse Dicke auf, besonders auf dem Quersehnitt. Auf den ersten Blick könnten solche Primitivbündel als hypertrophirte aufgefasst werden, wie sie bei verschiedenen nervösen MuskelAffectionen von einigen Autoren beschrieben warden (Cohnheim, Knoll, Scbultze u. s. w); bei genauerer Untersuchung aber erweisen sie sich als Muskelprimitivbündel, welche leicht aufgequollen oder viel weniger atrophirt sind, wie die benachbarten; denn bei den ersteren sind die Querstreifen und die Cohnheim'schen Felder sehr mangelhaft geworden, und bei den letzteren äberschreitet die Muskeldicke nicht das absolute Maass des physiologischen Verhältnisses (die dicksten Muskelprimitivbündel messen im Querdurchschnitt $40-55, \omega_{\text {) }}$.

Sehr auffallend und höchst interessant ist die Veränderung der Kerne, worauf ich speciell meine Aufmerksamkeit gelenkt habe. Sie fällt in directer Näbe der leprösen Heerde am meisten auf, während die weit abgelegenen, makroskopisch normal aussehenden Stellen dieselbe Veränderung zeigen, aber nur in sehr geringem Maasse. Als ich nun jene Stellen mit schwacher Vergrösserung untersuchte, so zeigte sich sofort ein sehr eigenthüm- 
liches Bild: die Kerne des Muskelgewebes sind im Ganzen vermehrt, vergrössert und unregelmässig angeordnet; sie haben ausserdem eine intensive Tinction mit allen Kern-färbenden Mitteln angenommen (Fig. 2). Bei eingehender Untersuchung mit starker Vergrösserung unter Anwendung feinerer Untersuchungs-Methoden ergiebt sich ein äusserst merkwürdiges Verhalten der Kerne, welches den Untersucher sowohl äberrascht, als auch oft verhindert, zu einer richtigen Erkenntniss der Dinge zu kommen. In Bezug auf Beschaffenheit, Gestalt, Grösse und Lage weichen die Kerne auffallend von der Norm ab und sind sehr verschieden von einander. Trotz ihrer Mannigfaltigkeit glaube ich die Kerne in folgende Kategorien ordnen za können, wenn auch Uebergangsformen zwischen allen Typen vorkommen.

I. Die Muskelkerne, sowohl die mitten im Muskelprimitivbündel, als auch die dicht unterhalb des Sarcolemma gelegenen, normal in Form und Grösse, vermehrt in ihrer Zahl, sind durch alle Kern-färbenden Mittel diffus und stark zu färben, so jedoch, dass sich auch bei sehr starker Vergrösserung keine deutlichen Kerngerüste wahrnehmen lassen (Fig. 3). Manchmal ist die Kernwand hyperchromatischer, als das Innere. Solche Kerne bilden entweder einen Haufen von länglich ovaler Gestalt oder eine lange einfache Reihe, einen "Zellkernstrang". Die Muskelprimitivbündel, in welehen diese Kerne sitzen, sind immer atrophisch, ihrer deutlichen Streifung beraubt, und manchmal ganz körnig zerfallen oder von zahlreichen Pigmentkörnern durchsetzt. Zuweilen bieten die Muskelprimitivbündel eine ampulläre Atrophie dar, und in den eingeschnürten Stellen treten solche Zellkernhaufen mit einer körnigen Masse auf. Diese Art der Kern-Veränderung (Vermehrung und Hyperchromatose) erinnert an sog. "Wucheratrophie", wie sie bei verschiedenen, sowohl allgemeinen, als auch localen Processen beobachtet wird, z. B. an den Muskelprimitivbündeln in der Peripherie der Geschwülste ${ }^{1}$ ); im jetzigen Fall ist aber diese Wucherung nur in viel geringerem Grade und an wenigen Stellen vorhanden. Manchmal scheinen diese Kerngruppen ausserhalb der Muskelprimitivbündel und zwischen denselben gelegen zu sein, indem sich keine typische

1) Siebe meine Arbeit: Ueber das histologische Verhalten des quergestreiften Muskels an der Grenze bösartiger Geschwülste. 
quergestreifte Nuskelsabstanz um die Kerne her nachweisen lässt. Dass aber die Kerne immer mit kömiger, pigmenthaltiger Masse umgeben sind, wie sie sonst bei degenerirenden Muskelprimitivbündeln beobachtet wird, weist mit grösster Wahrscheinlichkeit auf ihre musculäre Natur hio. An wenigen Stellen sind solche Kerne so dicht zusammen gedrängt, dass sie wie ein einziger, sehr grosser, maulbeerartiger Kern aussehen; die genauere Untersuchung lässt sofort ihre wahre Natur constatiren, indem der Contour jedes einzelnen Kerns zum Vorschein kommt.

II. Die Muskelkerne der zweiten Kategorie zeigen sich vergrössert und hyperchromatisch; letztere aber nur in geringem Maasse. Die dicht unter dem Sarcolenma gelegenen Kerne sind unregelmässig rundlich oder polygonal gestaltet. Es lüsst sich im Gegensatz zur ersten Kategorie eine unregelmässige Figur der Chromatin-Substanz des Kerns wahrnehmen; manchmal enthalten die Kerne ein oder mehrere hellere Fleckchen, welche wahrscheinlich eine partielle hyaline Degeneration bedeuten (Fig. 4). Solche Kerne erschienen vereinzelt, häufiger aber in Gruppen; ist letzteres der Fall, so sind sie dicht neben einander gereiht, und lassen durch eine schmale, helle Linie ihren Contour erkennen. An anderen Stellen nehmen die ebenso beschaffenen Kerne eine ganz andere Form an, nehmlich die der einfachen Verlängerung, welche manchmal sehr bedeutend ist (bis $55 \mathrm{p}$ ), und bilden eine Kernreihe oder einen Zellkernstrang. Eine solche Kerureihe liegt gewöhnlich in der Axe des Muskelprimitivbändels, and zwischen einzelnen Kernen befindet sich eine geringe körnige Masse, während das übrige Protoplasma des betreffenden Muskelprimitivbündels mit wohl erhaltener Querstreifung versehen ist. (Fig. 5). Der Name "Muskelkörperchen-Stränge" dürfte in diesem Falle auch nicht unpassend sein. Eine ähnliche, lange, strangartige Form können die unter dem Sarcolemma gelegenen Kerne, welche ebenso beschafien sind, auch dadurch erlangen, dass sie sieh vermehren, einfach verlängern, und dicht hintereinander in gerader Richtung wachsen; manchmal ist die Grenze einzelner Kerne sehr undeutlich, sogar kaum erkennbar, so dass der Gedanke nahe liegt, es sei eine Verschmelzung der Kerne eingetreten. Kerne dieser Kategorie, besonders der zuerst erwähnten Form, können eine 
bemerkenswerthe Modification zeigen, nehmlich „Fortsatzbildung" (Fig. 6). Ein langer, sich allmählich zuspitzender, dünner Fortsatz entwickelt sich aus einer unter dem Sarcolemma liegenden, sehr dichten Kerngruppe, indem ein Kern in derselben diese eigenthümliche Gestalt annimmt. Der Fortsatz steht durch eine breite Basis mit der Kerngruppe in Verbindung und endet entweder allmählich zugespitzt oder, wenn auch selten, mit einer kleinen, keulenartigen Auftreibung. Er kann sich aber in anderen Fällen mit dem gleichartigen einer anderen Kerngruppe verbinden. Die Vereinigungsstelle beider Fortsätze ist nicht immer zu bestimmen.

III. Zu einer anderen Kategorie gehören die Kerne, welche sich durch eine starke Hyperchromatose und eine starke Grössenzunahme auszeichnen. Der Recklinghausen'sche Satz ${ }^{1}$ ): „äusserst grosse, kernartige, namentlich mit Carmin stark färbbare Körper" bezieht sich auf diese Kategorie. Ich möchte hier noch mehrere Unterarten unterscheiden. Eine Form kennzeichnet sich dadurch, dass die Kerne ungefähr 3-5 mal grösser sind, als ein gewöhnlicher Muskelkern, sich wie ein dicker und langer Klumpen oder wie eine kurze Stange gestalten, entweder mit glatter Wand, oder mit unregelmässigen Ausbuchtungen versehen sind, und sich mit allen Kern-färbenden Mitteln stark und gleichmässig tingiren. Sie sind manchmal zwischen relativ wohl erhaltenen Muskelprimitivbündeln eingebettet, so dass der Gedanke nahe liegt, sie seien nicht den Muskelprimitibündeln selbst angehörig, sondern dem Interstitium. Bei der Untersuchung zahlreicher Präparate habe ich zuweilen aber mit Sicherheit denselben Körper innerhalb der Muskelprimitivbündel gesehen, wie der Querschnitt der letzteren am deutlichsten lehrt (Fig. 7). Der Kern liegt entweder gerade in der Mitte des atrophirten Muskelprimitivbündels oder an dessen peripherischer Partie; die ihn enthaltenden Muskelprimitivbündel, meist stärker verändert als die anderen, sind entweder einfach atrophisch oder körnig degenerirt; nur einmal habe ich einen solchen Kern in einem relativ wohl erhaltenen Muskelpimitivbündel gesehen. Die scheinbar frei zwischen den Muskelprimitivbündeln liegenden Kerne sind auch mehr

1) a. a. 0. 
oder weniger von einer körnigen Masse, welche dem degenerirten Muskel-Protoplasma gleicht, umgeben. So könnte mit Recht der Schluss gezogen werden, dass diese grossen, klumpenartigen, scheinbar den Muskelprimitivbündeln nicht angehörigen Kerne doch eigentlich noch Muskelkerne sind. Ich habe diesen Klumpenartigen, hyperchromatischen Körper ohne Weiteres "Kem ${ }_{3}$ genannt; aber es könnte die Frage zunächst aufgeworfen werden: ob dieses Gebilde ein richtiger Kern oder ein anderer, nur färberisch sich wie ein Kern verhaltender Körper ist? Der Form und Beschaffenheit nach erscheint derselbe wesentlich anders, als ein gewöhnlicher Muskelkern; aber wenn er mit Sicherheit innerhalb des Muskelprimitivbündels zu beobachten ist, und zwar an der Stelle, wo sich gewöhnlich die Muskelkerne befinden, so darf ich ihn wohl mit Recht als einen veränderten Kern auffassen. Die andere Frage wäre: ob jene Kerne durch Vergrösserung aus einem Kern entstanden sind, oder ein Conglomerat mehrerer Kerne darstellen. Während ein bezüglicher Kern der ersten Kategorie bei stärkerer Vergrösserung deutlich die Contouren der einzelnen Kerne noeh orkennen lässt, und sich somit als ein Conglomerat erweist, ist der grosse, jetzt zu besprechende Kern immer ganz gleichmässig gefärbt und zeigt nirgends eine Andeutnng einer Zusammensetzung aus mehreren Körpern. Einige Kerne dieser Art, welche sich innerhalb eines wohl erhaltenen Muskelprimitibündels befinden, bieten manchmal eine modificirte Form dar, indem sie ungleiche Fortsätze besitzen oder eine unregelmässig strahlige Figur bilden (Fig. 8). Die ganze Gestalt erscheint ganz anders, als bei der zweiten Kategorie, insofern als der Fortsatz immer dick und kurz, ferner gleichmässig und auck stärker gefärbt ist. Diese Art der Metamorphose macht sie vielmehr derjenigen Form sehr ähnlich, welche Schmaus und Albrecht) in ihrer Studie über KernDegeneration an den Nieren-Epithelzellen als "Sprossenfigur" bezeichnet haben.

Eine weitere Species dieser Kategorie zeichnet sich durch ihre bedeutende Grösse und eigenthümliche Gestalt aus - die "Riesenkerne" (Fig. 9); dieselben sind hyperchromatisch, aber

1) Schmaus und Albrecht, Ueber Karyorhexis. Dies. Arch. Bd. 138. Supplementheft. S. 1. 1895. 
nicht so stark nnd gleichmässig, wie bei der zuletzt erwähnten Form, und sehr flach, statt klumpig, - wie dort —, breit, länglich abgerundet oder unregelmässig viereckig und meist so, dass ihre der Längsrichtung der Muskelprimitivbündel entsprechenden Ränder gekrümmt sind. Bald überwiegt der Chromatingehalt am Rande des Kernes, bei weitem der häufigere Fall, bald der in seinem Inneren; die meisten dieser Kerne sind ebenso, wie die der zweiten Kategorie, von hellen Flecken durchsetzt, und zwar treten diese gewöhnlich in einer Mehrzahl auf. Was die Lage solcher Kerne betrifft, so sind sie immer in der äussersten Schicht, anzutreffen. Oft lässt es sich sehr schwer entscheiden, ob der Kern dem Muskelprimitivbündel angehört oder dem Interstitium. Das Querschnitt-Bild und sorgfältig verfertigte ZerzupfungsPräparate der Muskelprimitivbündel lehren aber, dass sich wenigstens die meisten Kerne sicherlich innerhalb des Sarkolemma befinden. Am schönsten und sehr leicht orientirend ist das Bild, welches ich gewinne, wenn ich nach der Vorfärbung der Kerne die Querstreifen der Muskelprimitivbündel färbe. (Ich habe hierfür Methylenblau benutzt, kann aber die genaue Tecbnik noch nicht angeben, da das Resultat der Färbung nicht immer das Gleiche ist, wenn ich auch gelungene Bilder von sehr schöner Färbung erhalten habe.) Die Krümmung der Ränder bedeutet oft nichts anderes, als dass der der Fläche nach gebogene, der Krümmung des Muskelprimitivbündels angeschmiegte Kern auf dem optischen Durchschnitt gesehen wird. Ferner lehrt der Querschnitt der Muskelprimitivbündel, dass sich die Kerne nicht selten mehr als die Hälfte des ganzen Umfanges der Muskelprimitivbündel ausbreiten. Seltener bleibt der eine Rand des Kerns nicht an der Oberfläche des Muskel-Pr.-B., biegt von dieser vielmehr nach innen ab und dringt auf eine Strecke zwischen die Maskelfibrillen hinein (Fig. 10). Dieses Verhalten liefert auch einen Beweis dafür, dass der Kern dem Muskel-Pr.-B. angehört. Ich muss ferner hervorheben, dass die mit diesen Kernen besetzten Muskelprimitivbündel, im Gegensatz zu der ersten Kategorie, meist relativ kräftig erschienen und ihre Querstreifung noch erhalten haben. Sind nun derartige Riesenkerne durch Vergrösserung eines einzelnen Kernes oder durch Confluenz mehrerer Kerne entstanden? 
Die Antwort auf diese Trage fällt nieht immer klar aus. Sicherlich bekommt zuweilen ein einzelner Kern eine grössere Gestalt, indem seine Substanz eine Art Aufquellung erfährt and sich damit eine Hyperchromatose, sowie eine durch die punktartigen, hyalinen Flecken bezeichnete Degeneration verbindet; andererseits kann eine Art yon Zusammenfliessen mehrerer Kerne nicht minder deutlich constatirt werden. An dew einen Ende eines Riesenkernes sind nehmlich noch die Contouren verschiedener Kerne gut zu erkennen oder als feine helle Linien angedeutet, während das andere Ende des Kernes, abgesehen von einzelnen kioinen, hellen Flecken in seinem Inneren, eine bomogeno Beschaffenheit darbietet. In günstigen präparaten kann der allmäbliche Uebergang dieser beiden Partien verfolgt werden. Ich nehme deshalb zweierlei Entststehungsarten dieser Riesenkerne av. Oefters erhalten solche Riesenkerne eine eigenthümliche, unzusammenhängende Gestalt; die Kerne vergrössern sich nehmlich im Gamzen, treiben Aeste oder nehmen Zickzackform an, und sehen wie aufgelöst aus. Einige davon besitzen eine beträchtliche Grösse (88 $\mu$ oder noch mehr in dem Längsdurehmosser). Andere, und zwar aus vielen Kernen zusammengesetzte Körper tragen oft an ihrem Ende Haken oder bilden „wie Spangen umklammernde" Gestalten (v. Recklinghausen, a. a. 0. S, 11-12.) Diese wie Spangen umklammernde Form ist bei den anderen Kernen noch viel deutlicher ausgeprägt, wovon ich jetzt weiter reden will.

Derartige Kerne sind vergrössert, länglich gestaltet, stark hyperchromatisch, liegen quer oder schräg zar Axe des Muskelprimitivbündels und klammern sich an diese sogar. wie Haken oder Spangen an (Fig. 11). Ihre Lage ist meistens gleich der des Riesenternes, nehmlich in der äussersten Schicht eines Muskelprimitivbündels. Nur sehr selten befindet sich ein gleich gestalteter, quer gelegter, hufeisenförmiger Kern mitten in dem Muskelprimitivbündel (Fig. 11 c). Trotz des beträchtlichen Unterschiedes der Kerngrösse habe ich diese beiden Kern-Arten in die gleiche Kategorie eingereiht, da dieselben grosse Aehnlichkeit aufweisen, und da ich nicht selten Uebergangsformen zwischen beiden wahrgenommen habe. Die Hufeisenform wird entweder durch einen einzigen, oder durch 2 bis 3 zusammen- 
hängende Kerne hergestellt; nicht selten entsteht auch ein fast. geschlossener Ring, welcher sich um ein Primitivbündel schlingt. Eine ähnliche, aber dem Muskel-Primitivbündel parallel liegende hufeisenförmige Kernkette wird auch, allerdings selten, durch Kerne der ersten Kategorie gebildet (Fig. $3 \mathrm{c}$ ); aber durch die Beschaffenheit der einzelnen Kerne lässt sich davon die jetzt besprochene Form leicht unterscheiden. Eine Modification dieser Form zeigt ein langes, hyperchromatisches Gebilde, wie es die Fig. $11 \mathrm{~d}$ darstellt. In dem einen, kolbig aufgetriebenen Ende sind zahlreiche, noch intensiver gefärbte Körperchen eingebettet, welche der Grösse und Gestalt nach den Kernen der ersten Kategorie gleich kommen. Ob dieses Gebilde mit den anderen „umklammernden Kernen“ zu identiflciren sei, lässt sich schwer entscheiden. Am nächsten liegt es, anzunehmen, dass es sich um eine combinirte Form der zuletzt erwähnten Kern-Art mit derjenigen der ersten Kategorie handelt. An einem solchen Präparate blieb es mir auch manchmal uneotschiedon, ob das durch Hämatoxylin oder Carmin u. s. w. stark gefärbte Gebilde in allen seinen Theilen ohne Weiteres als Kern-Substanz zu betrachten ist, oder ob daran noch das modificirte MuskelProtoplasma Theil hat. Der Verdacht auf Verkalkung, welcher wegen der durch Hämatoxylin hervorgerufenen dunkelblauen Färbung erweckt wurde, konnte, da die mikrochemische Reaction negativ ausfele abgewiesen werden.

IV. Für eine ganze Reihe dieser umklammernden Formen muss aber, so sehr sie auch in ihrer Gestalt und in ihrem Aeusseren der dritten Kategorie der Muskelkerne ähneln, meiner Meinung nach eine andere Herkunft angenommen werden. Man erblickt nehmlich, wie Figur 12 lehrt, manchmal leicht vergrösserte und oblonge Kerne, welche schief oder quer zu den Primitivbündeln gerichtet sind und sich äber 2 Muskelprimitivbündel fort erstrecken, - ein deutlicher Hinweis, dass sie sicherlich den Muskelprimitivbündeln selbst nicht angehören. Manchmal ziehen sich solche Kerne deutlich an einem Capillargefäss entlang, welches schief auf den Muskelprimitivbündeln liegt (Fig. 12b). Auch zeigt ein die Muskelprimitivbündel umklammernder Kern znweilen deutlich die Höhlung im Inveren, welche dem Lumen eines Capillargefässes entspricht. In eivigen 
Stellen habe ich in diesem Lumen eines solchen Kerns viele Mikrokokken aufgefunden, denen wohl keine Bedentung beizumessen ist, aber durch welche das Vorhandensein eines Canals mit grosser Sicherheit constatirt werden konnte. Häufig sind derartige Kerne auch mit Verzweigungen versehen. Ich halte daher Kerne dieser Art für Endothelkerne der Blat- and auch der Lymphgefässe, obgleich letztere ja im Muskelgewebe nur schwach entwickelt sind. Die v. Recklinghausen'sche Beschreibung: "Da sie (äusserst grosse, kernartige, namentlich mit Carmin stark färbbare Körper) bisweilen längliche Gebilde darstellten und sogar kurze Zweige trugen, so durfte man wobl an Zellkernstränge denken, and sie von Gefässwandungen, von veränderten Endothelien der Blut- und Lymphcapillaren herleiten ", ist für derartige Kerne gewiss zutreffend. In Serienschnitten konnte ich zuweilen feststellen, dass solche zwischen den Muskelprimitivbändeln gelegenen Kernstränge einen dem Gefäss entsprechenden Verlauf nahmen. Zaweilen bekam ich den Eindruck, dass sich an der Bildung des ganzen Stranges nicht bloss die hyperchromatisch gewordenen Kerne betheiligen, dass er sich vielmehr aus den veränderten Gefäss-Endothelien selbst zusammensetzt, während die darin eingeschlossenen intensiver gefürbten kleinen Gebilde als die Kerne der Endothelzellen angesprochen werden müssen. Meine oben für die dritte Kategorie ausgesprochene Ansicht, dass nïcht nur der Kern, sondern auch das Protoplasma selbst eigenthümlich verändert sei, findet hier wenigstens eine Stütze.

Es sind bei dem hier berührten Leprafall hauptsächlich die Veränderungen im Maskelgewebe, welche mich interessirt haben, vor Allem die Veränderung der Kerne, indem diese am leprös erkrankten Muskel noch nicht beschrieben war, bis Herr Prof. v. Recklinghausen sie in der Sitzung der pathologischen Geselischaft demonstrirte. Ueberhaupt ist aber eine derartige Kern-Veränderung des Muskelgewebes in der Literatnr, so weit ich in derselben naohforschen konnte, nicht angegeben. Nur

) a. a. $0 .$, S. 12 .

2) Askanazy, Pathologisch-anatomische Beiträge zur Benntniss des Morbus Basedowii u. s. w. Deutsches Archiv f. klin. Med. Band 61, S. $118,1898$. 
hat neuerdings Askanazy ${ }^{2}$ ) in seinem III. Fall von Morbus Basedowii (bei einer 5ljährigen Frau, welche länger als sechs Jahre eine Hals-Anschwellung hatte) ausser der interstitiellen Lipomatose des Muskelgewebes verschiedene Muskelkern-Veränderungen: "Die Confluenz der Kerve", „dicke Klumpen“, "platte Bänder, die sich um einen Faserabschnitt herumschlingen", „dachrinnenartig gebogene Kernplatten " beschrieben und abgebildet; diese Kern-Veränderung stimmt in den meisten Punkten mit den von mir beschriebenen überein, so dass man sie mit grösster Wahrscheinlichkeit auf die gleiche Stufe mit unserer Kern-Veränderung stellen kann, mag letztere auch vielleicht ausgeprägter erscheinen und zahlreicher sich nachweisen lassen, wie in dem Fall von Askanazy. Mit Recht hat letzterer diese eigenthümlich veränderten, zuweilen scheinbar ausserhalb der Muskelprimitivbündel liegenden Kerne als Muskelkerne betrachtet. Auch in meinem Fall sind die meisten wohl innerhalb der Muskelprimitivbündel entstanden oder durch den Zerfall derselben blossgelegt worden; aber ein Theil der eigenthümlich veränderten Kerne gehört dem ausserhalb der Muskelprimitivbündel gelegenen Gewebe an und ist den Gefäss-Endothelien entsprossen.

Sind nun diese Kern-Veränderungen, wie ich schon erwäbnte, in der directen Nähe des leprösen Herdes besonders ausgeprägt, so tragen die Muskelkerne an weit entfernten Stellen dieselbe Veränderung gar nicht oder nur andeutungsweise. Ferner muss ich aber bemerken, dass die veränderten Kerne mit den Leprabacillen keineswegs in unmittelbarer Berührung stehen, und ich muss daher die Möglichkeit ausschliessen, dass diese Kern-Veränderung durch eine directe Beeinflussung seitens der nachbarlich aufgetretenen Leprabacillen hervorgebracht worden sei. Ich habe mich auch bemüht, den feineren Verlauf der inter- und intramusculären Nerven-Elemente zu verfolgen, um zu untersuchen, ob ihre Erkrankung irgend eine aetiologische Bedeutung für die Kern-Veränderung hätte. Nirgends aber konnte ich constatiren, dass diese Kernveränderung mit der Nervenfaser-Entartung, welche überhaupt unbedeutend und inconstant erschien, in etwaiger directer Beziehung stand. Was die Nervenendigung betrifft, so konnte ich, da das in Formalin gehärtete Präparat für deren Nachweis nicht geeignet vorbereitet war, 
trotz aller angewandten Mühe keine schônen, brachbaren Präparate erzielen; doch bin ich wenigstens za der Ueberzeugung gelangt, dass die veränderten Kerne aller Arten nichts mit der Eintrittsstelle der motorischen Nervenfaser in das Muskelprimitivbuindel zu thun haben.

Die besprochene Veränderung des Muskelgewebes wird also direct weder durch die Leprabacillen, noch dureh die Nervenfaser-Entartung hervorgerufen; ich sehevielmehr darin eine Wirkung der von den leprösen Heerden bedingten collatateralen Saftstrom-Aenderung auf das Muskelgewebe, d. i. eine directe Storung seiner Ernährung, welche hauptsächlich an den Muskelkernen, theilweise auch an den Gefäss-Endothelkornen, und höchstwahrscheinlich auch an den Gefäs-Endothelien selbst zum Ausdruck gelangte.

\section{Erklärung der Abbildungen auf Tafe! V.}

Fig. 1. Leprazellen und freie Leprabacillen im Interstitium des Mluskelgevebes: Grain'sche Färbung nach der Kern-Yorfärbung wit Carmin. (Zeiss, II. AA. Oelimersion $1 / 12$ ).

Hig. 2. Muskelgewebe nahe dem leprösen Heerde der Haut. Vermehrung, Vergrössernng, ungewöbnliche Gestaltung aud starke Tingirbarkeit der Kerne sebr auffallend. (a) körnige Zerfallsmasse im Interstitium mit Leprazellen. Schwache Vergrösserung (Zeiss, II. AA.)

Fig. 3, a) b) c) gewueherte, stark und diffus hyperchromatische Muskelkerne in normaler Grösse. I. Categorie. (Zeiss IV. DD.)

Fig. 4. Grosse, polygonale, unter dem Sarcolemma liegende Muskelkerne, nur leicht hyperchromatisch und mit bellen Fleckchen versehen. il. Kategorie. (Z eiss IV. DD.)

Fig. 5. Lange, mit bellen Fleckchen versehene, leicht hyperchromatiscbe Muskelkerne, welche sich der Achse des Muskelprimitivbündels entlang aufstellen. II. Kategorie. ( $\mathrm{Z}$ eiss IV. Oel-Immersion 1/12).

Fig. 6. $Z$ wei durch lange und schmale Fortsätze verbundene Kernbaufen in einem Muskelprimitivbündel. II. Kategorie. (Zeiss IV. DD.)

Bigg. 7. Querschnitt eines Muskelprimitivbündels. Hypercbromatischer, grosser, klumpenartiger Kern in der Mitte des Primitivbündels. 111. Kategorie. (Z eiss IV. DD.)

big. 8. a) b) sprossenartig gestalteter, stark hyperchromatischer Muskelkern auf dem Querschnitt eines Muskelprimitivbündels. III. Kategorie. (Zeiss IV. Oelimmersion $1 / 13$ ). 
Fig. 9. a) b) c) verschiedene Formen der Riesenterne. III. Kategorie. (Zeiss IV. Oel-Immersion 1/13).

Fig. 10. Riesenkern mit einem nach Innen eingeknicktem Rand. Querschnitt eines Muskelprimitivbündels. III. Kategorie. (Z eiss IV. Oelimmersion $1 / 12$ ).

Fig. 11. a) b) c) d) verschiedene Formen der ein Muskelprimitivbündel umklammernden, bezw. hufeisenförmigen Muskelkerne. III. Kategorie. (Zeiss IV. DD.)

Fig. 12. a) b) ebenso Mustelprimitivbündel umblammernde oder äbulich gestaltete Kerne und Gefâss-Endothelien (b).

c) d) byperchromatische Gefäss-Endothelkerne. IV. Kategorie. (Z e is s IV. DD.)

\title{
VIII.
}

\section{Untersuchungen über die Mechanik der Expectoration.}

\author{
Von
}

Prof. Dr. Geigel in Würzburg.

(Hierzu 3 Text-Abbildungen.)

Der Mechanismus der Athmung scheint in allen seinen Theilen hinreichend erforscht zu sein. Man kennt die inund die exspiratorischen Muskeln, den negativen und den positiven Druck, den sie im Thorax durch ihre Thätigkeit erzeugen können, bei ruhiger Athmung sowohl, als auch bei maximaler Anstrengung. Bekannt ist auch das Quantum Luft, das in beiden Fällen ein- und ausgeathmet wird. Damit ist Alles gegeben, was vom mechanischen Standpunkte aus physiologische Bedeutung für den Gaswechsel in den Lungen hat.

Eines ist aber, so viel ich sehe, noch nie beobachtet und bestimmt worden, das ist die Geschwindigkeit, mit der die Luft in den Thorax gezogen und bei der Exspiration ausgeśtossen wird. Diese ist physiologisch wichtig für das Anblasen der Stimmbänder, für die Bildung der Stimme und Sprache und, was für den Pathologen und Kliniker von Bedeutung wird, 